\title{
Creativity and Dysfunction in Strategic Processes: \\ The Case of Scenario Planning
}

\section{Peter McKiernan, University of St Andrews}

\subsection{Introduction}

Creativity and innovation, like entrepreneurship, are projected in the management literature as unequivocally wholesome and appealing. Clearly, in certain contexts e.g., corporate or sector ossification, their stimulus to change management is necessary and acknowledged widely. Each has a strong and influential role in the facilitation of constructive conflict and as a trigger for improving employee attitudes and emotional well-being. Logically, the theoretical prognosis for performance from any process engaged with them is positive and recurring. But, there is a more disturbing aspect to this pairing, revealed often in empirical work [1], whose existence remains covert and whose effects can be damaging e.g., the destructive effects to pride, value systems and motivation. Scarcely is this shady aspect recognised in mainstream research or teaching so reinforcing the prejudice of endless, bountiful returns.

The purpose of this paper is to begin the exploration for such dysfunctional effects in a foresight process renowned for its creative and innovative ingredients and experiences, and one that has been adopted widely in recent years by nations, organisations and individuals-scenario planning. Although 'pitfalls' of the scenario planning process have been identified previously [34], these tend to be generic obstacles to getting a 'good job done' rather than a deep, investigative focus on the potential dysfunctions of the 
'creative' aspects of the process. To achieve this objective, the paper is structured into

four sections. The first section of the paper examines the creative and innovative process as depicted, in the main, by social psychologists concerned with managerial productivity and human performance and spirit. Definitional issues are discussed and the psychologist's emphases are informed by literature from the creative (or cultural) industries on the nature of the creative process and its constituent elements. The second section recognises the rich arsenal of scenario planning processes and focuses upon those with a deep heuristic platform underpinned by 'disciplined intuition' or 'intuitive logics' [2-5]. Here, the creative credentials of the process are assessed by comparison with the creativity definition and nature of the creative process illustrated in section one. The third section examines the scenario process stages in detail and explores the potential dysfunctions of the creative aspects of the individual stages and of the process as a whole. The concluding section reflects on the impact of this 'darkness' and offers suggestions for process improvement.

\subsection{Creativity and Innovation}

\subsection{Definitions of creativity and innovation}

Definitional dialogue in the management, psychology and 'creative' industries literatures has raged long and sharp over each of the terms 'creativity' and 'innovation' and over the distinction between them, with no emergent consensus. In received management discourse, definitions of creativity have emphasised both individualism, as in the isolated, mythical genius, and novelty, as in the path breaking idea or invention. Bilton [6] argues 
that this 'conflation' to the individual and innovation, with its pathology in Western philosophy, reinforces a one-sided stereotypical view of creativity and creative people as it disconnects them from the context that fertilises their ideas and talents and so gives them meaning and value. Consequently, by idolising individuals over the creative process, 'we start to build ... systems of management and organization which are at odds with the real needs of creative people and processes' [6, p. 7]. Definitions in psychology are seen as superior to those in management as they hint at a 'duality' of novelty and utility with deference to the context in which the creativity occurs.

In social psychological research, West and Farr [7] have provided one of the most respected definitions of workplace innovation:

"...the intentional introduction and application within a role, group or organisation of ideas, processes, products or procedures, new to the relevant unit of adoption, designed to significantly benefit the individual, the group, the organisation or wider society" (p. 9).

First, the definition distinguishes between the two variables, with innovation embodying both intent and process improvement, and with creativity, defined in absentia, left to refer solely to idea generation. This creates what King [8] has referred to as an 'ideationimplementation dilemma'- the generation of original ideas and the complexity of their metamorphosis into practice.

Second, as Anderson and Gasteiger [9] point out, innovation, rather than creativity, must provide quantifiable benefits to at least one of several stakeholder groups both internal and external to the organisation. Hence, innovative ideas must be 'fit and beneficial for purpose'. Third, innovation focuses on novelty relative to existing practice and knowledge [P-creativity in Boden [10] terms], allowing for the import and export of innovation from 
other work groups. Both the relative and absolute dimensions of innovation are context specific i.e., what is new to one group in organisation ' $x$ ' may be de rigour in organisation ' $y$ ' and what is new to organisation ' $z$ ' may not be known elsewhere in the world. Fourth, central to the definition is the considerable benefit to be delivered by innovative ideas to the many stakeholders. Any deleterious effects of these ideas or conflictual outcomes of process implementation are either absent or anonymous. This restricts the definition to innovations that have wholly positive effects and, arguably, renders it solely a theoretical definition that is impossible to embody in practical observance.

For the purposes of this research, we adopt the directed, process- based definition provided by West and Farr [7] where both creativity and innovation are embraced as integral parts of a specific or continuous process that contains ideas or actions of novelty and utility but adapt it by allowing for adverse or surprising, unintentional outcomes. Contrary to managerial prescriptions of 'brainstorming', lateral thinking [11] and 'thinking outside the box', Bilton [6] argues that this duality of creativity emerges from the combination of different types of thinking, both convergent and divergent. As in a combination of left and right brain activities, creativity occurs at their intersection bounded within an envelope of 'conceptual space'. This space is not too close to conventional systems and thinking in the organisation where it will be absorbed rapidly but lack novelty, and not too far away that there is too much novelty for the system to digest-the creativity should surprise but not shock. This creative envelope follows Boden's [10] notion of 'boundary tweaking'-at the edge of chaos and order. Noting that 
such tweaking involves a combination of stretching and retaining forces, we turn our attention to how these features manifest themselves in a creative process.

\subsection{Creative Processes -Psychology}

In general, the exposition of creative or innovative processes by academics has been based upon a traditional, stage based, Input-Transformation-Output (ITO) sequence (see the multitude of variants in [12] and Figure 1). Specifically, individual, group or organisational characteristics (e.g., cognitive ability, personality, motivation, charisma, knowledge, norms, size, culture, rewards) act as antecedent variables that condition the quality and extent of creativity that, in turn, determines the quality and extent of innovation and that, in turn, produces a variety of outcomes at a particular level of analysis (see, for instance, $[13,14])$. Developmental adaptations of the process include the introduction of moderating variables (e.g., voice behaviour, personal initiative-see [15]) between the stages that influence the generation of novel and useful ideas, their implementation into new products or processes and their assistance in the prediction of outcomes. Conventionally, these outcomes have assumed a positive hue. For instance, in terms of groups, these include successful innovation, group cohesion, group potency, clear objectives, clear leadership, group effectiveness and receptivity to future innovation projects.

\section{$<$ Figure 1 about here $>$}

The exploration of the moderating variables in the ITO process has allowed investigations into its benefits and costs $[9,16,17]$. Negative outcomes include discontent and low performance [18], theft, sabotage and harmful behaviour [16], destructive conflict, 
innovation failure, negative job attitudes and stress [17], employee burn out, privacy and security problems [19] and lower efficiency of work processes [20]. Ironically, such elements (e.g., sabotage, destructive conflict) form part of the creative phase of innovation programmes in terms of idea generation and 'thinking better inside and beyond the box'. As Anderson and Gasteiger [9] note:

"Behaviour by individuals that challenges established practices that violate existing rule structures, or that involve the misuse of organisational resources for non-sanctioned activities is potentially understood as being counterproductive...yet...all are often present in early innovation attempts." ${ }^{1}$

Clearly, stage based models have their critics (see [12]), for instance, in terms of linearity, practicality, precision and integration. But their reductionism allows individual phases to be examined for function and dysfunction in a way that would be difficult to source over a total process. Research in the cultural industries [6] [24] follows a similar structural patterning but emphasises the integration of the phases as a whole process and not their isolation where they might become victims of individual specialisation, division of labour and analysis of a scientific management nature.

\subsection{Creative Processes- Cultural Industries}

Within the cultural industries, the creative process emphasises the combination of idea generation and the systematic application and hard graft that accompany it. In the myth of genius, Weisburg [23] showed how the masterly contributions of Mozart, Picasso and Coleridge could be explained. These occurred not simply by a spark of creative genius but

\footnotetext{
1 The literature on counter productivity in workplace behaviour (see [21]) seems to be a remarkable reciprocal of that on workplace innovation e.g., the notion 'skunkworks' as creative vehicles in corporations [22].
} 
by logic, memory, chance, training and long hours of application. The inventor, Thomas

Edison, described it so:

"Genius is one percent inspiration, ninety nine percent perspiration."

Hence, in any creative process, these two elements have to be integral. Bilton [6] has used Poincare's [24] four steps of preparation, incubation, illumination and verification (see Figure 2) within the cultural industries to good effect. The model combines and juxtaposes left and right modes of thinking; the creative with the conservative, as artists and writers spend long periods under both forms of thought before a work bears fruit. Under preparation, individuals or groups scope the issue under investigation, define the boundaries of the project and gather and interpret existing knowledge. Incubation involves the absorption, reflection and massaging of the issue at a sub-conscious level until illumination marks the ecstatic (Eureka) moment when the mosaic elements of the issue emerge innovatively into a meaningful pattern. This outcome is subjected to verification against the original issue, new data or practical application to prove its utility. By embracing both novelty and utility, Poincare's model reflects the psychological definitions of creativity in section 2.1 above. Bilton [6] advises:

"In the management literature on creativity, the duality, paradox and contradictions of creativity are frequently acknowledged and alluded to. But, where I believe the psychological theories of creativity, and Poincare's model in particular, represent an argument for greater thinking styles and competencies, the management literature tends to use these models to argue for greater specialisation and separation." [6, p. 11]

\section{$<$ Figure 2 about here $>$}

With a good sense of the definition of creativity and what constitutes a creative process, we turn our attention to the examination of scenario planning. Often, facilitators and 
participants perceive this process as both creative and innovative yet it is subjected rarely to a detailed appraisal of its creative elements. The next section introduces the process and assesses its claim to be creative given both the definitions in section 2.1 above and the nature of creative processes in 2.2 and 2.3 above. We investigate the question less asked in the strategy community-is scenario planning a creative process? If it proves so, it will be a useful vehicle with which to investigate the dysfunctions associated with creativity.

\subsection{The Scenario Planning Process}

\subsection{Historical Development}

Scenario planning is an intervention used within the strategic management of resources that combines the generation of stories, images, collage or poems of plausible, alternative future environmental states with the practical strategic and policy means of providing for them. The scenario planning process (SPP) has a special benefit in making sense of contexts rich in rapid change and complexity where the traditional forecasts of statistical modelling struggle for accuracy. Scenario processes vary widely and range from the highly quantitative, based on mathematical probability and computer simulation, to the highly qualitative, based on the creation of literal and visual imagery.

The contemporary history of these processes is rooted in the distinctively different American and European commercial contexts, yet their origins are profound, namely US 
military planning during WWII and the post war rebuilding of Europe, especially France ${ }^{2}$.

Concerns among senior US commanders over the link between military strategy and

technological development during WWII, and the unlikely prospects of long-term peace

thereafter, led to the establishment of the RAND Corporation in 1945 as a conduit for

applied futures research. By the 1950s, its 'systems theory' approach was extended from

defence studies into social issues like urban decay and resilient poverty. One of its most

influential thinkers, Herman Kahn, migrated to set up the Hudson Institute in 1961,

where his foresight studies imagined the 'World of 2000'. These future conjectures were

based primarily upon the analysis of long-term trends e.g., population growth, and their

impact at key stages in societal development. After Hollywood, he coined the term

'scenarios' 3 for the resultant future landscapes and defined them as:

"A hypothetical sequence of events... for the purpose of focusing attention on causal processes and decision points. " $[2,:$ p 6$]$

Kahn's research at Hudson attracted a variety of multi national companies including

Corning, IBM and Shell. The latter is credited often with the first commercial application

\footnotetext{
2 In post-war Europe, scenario planning emerged from the need to galvanise society and re-create cultures and economies from the grassroots. In France, Gaston Berger founded the 'Centre d'Etudes Prospectives' (CEP), where he developed a scenario approach to long range planning entitled 'La Prospective'. Berger believed that the future was not predetermined, but rather something that could be shaped to the benefits of wider society by emphasising positive future scenarios. By the 1960s, this approach had embraced education, the environment, urbanisation and regional planning, with Pierre Masse (the Commissioner for the French National Plan) incorporating the approach into the 4th French National Plan (1960-1965). Subsequent iterations of the CEP methodology focused on influencing key political groups and specifying ways in which these futures could improve the lives of ordinary people [25], and the development of fresh tools and techniques, including pioneering computer simulation, after the arrival of defence analyst Michel Godet in the 1970s.

3 The term 'scenarios' was used already in Hollywood for the production process leading to the generation of a complete script and the shooting directions of a motion picture. Well aware of this parallel, Kahn stressed nonfictional films in his analogy, as these were the basis for the imaginative stories he had in mind for describing possible futures. Ironically, in 'thinking the unthinkable' about thermonuclear warfare, the Dr Strangelove character in Stanley Kubrick's 1963 film of the same name was based on Kahn.
} 
of scenario planning ${ }^{4}$. Shell embraced a heuristic approach encapsulating ideas generation and future imaging, designed to challenge the mental mind-maps of process participants. These stakeholders come from all walks of life e.g., knowledge experts, consumers, owners etc., with share with each other their different lenses on the project under study. Once this is scoped, extensive data research follows on the main identifiable drivers of future change. Subsequent ordering and classification leads to an embracement of the uncertain elements, around which the scenarios are created, and how these might unfold over the medium to long term. Uncertainty comprehension requires more intuition, imagination and creativity than the understanding needed for variables with pre-determined outcomes e.g., demographics. This represents a stark challenge to cognition, especially for participants to whom this is a new way of thinking i.e., those with a narrow specialism (e.g., cost accounting) or with routine based, daily work. Often, their mental inertia needs considerable stimulus before they can 'think outside of the box'.

The resultant scenarios are tested rigorously e.g., for consistency, surprise, plausibility and gestalt. Iterations follow until a final version is produced that is acceptable to the tests, the participants and the main agent e.g., client executive. These stories of the future may metamorphose into film, artwork, collage, poetry or other media to increase their impact, as they inform strategy and policy. The primary difference between such a people centred, heuristic method and competing foresight methods is they do not rely on

\footnotetext{
${ }^{4}$ In 2000, the US Hart-Rudman Commission [27] investigating "American Security in the $21^{\text {st }}$ Century" were concerned that future studies failed to pick up soft signals that caused startling outcomes later. They explored 20 scenario studies and found 70 percent were surrogates of the Shell 'methodology'.
} 
mechanistic, formalized procedures, but on a flexible and diverse set of tools and techniques that actively encourage creativity and enable customisation to client, content and time dimension.

These scenario approaches are useful on several levels, since they: provide assistance in making sense of complex contexts making early strategy preparation possible; facilitate the rehearsal of the impact of structural and 'unknowable' variables on strategy; perform judicial duties in due diligence situations e.g., take-over, merger; aid flexible deployment of resources; prevent cognitive freezing on particular ways of doing things; unify teams around common goals; stretch collective ambition; generate new ideas and innovations and help solve seemingly intractable problems between fractious groupings, in a politically and philosophically 'neutral' space. However, perhaps the greatest contribution of the heuristic scenario planning method lies in its active engagement with stakeholders in a strong process and its power to enable them to think about complexity and uncertainty, and how they might shape the external environment to contribute to their strategic ends.

Critically, these processes are not without weakness. The 9/11 Commission [28] blamed the failure to spot the surprise attacks on a lack of imagination in scenario studies, despite the availability of warning signals. True, many projects failed to spot weak signals due to a common focus on popular elements such as global economics, the information revolution etc. But, this mimicry may have less to do with path dependency through a Shell- derived heuristic process and more to its poor facilitation or the dominance of commissioning agents within the process and over its outcome. Second, many general 
scenarios have been criticized for being too hierarchical, individualist and western biased, thus limiting their acculturation. White, male, middle-class stakeholders, often with shared value systems that disable or ignore competing worldviews, can dominate the scenario generation process. Third, as the process tracks trends from the past, through the present to the future, it has been argued that perceptual errors in conceiving of history e.g., as limited by national school curricula, can cause fatal flaws in the resultant scenarios [29]. Yet scenario planning, properly construed, retains superiority over forecasting based techniques for gazing into the medium and long term and obtaining a 'feel' for how things might shape up and how they might impact on particular situations. Thus, strategy and policy become better informed.

\subsection{The Architecture of a Heuristic Scenario Planning Process}

The generic architecture of a heuristic based SPP is displayed in Figure 3. While retaining a strong linear structure, the process is multi staged, interactive, iterative and data driven. The strong structure is seen as critical to restrain the many 'messy' diversions of thought and dialogue that could stretch and de-rail the process otherwise. The process has no specific time limits. Concise processes, for example as organised in an urgent reaction to organisational shock or crisis, can be accomplished in a couple of days while processes that explore complex topics, for example the future of a nation, have to be crafted carefully and can take a couple of years to complete. The interactive involvement of individuals and groups at different stages means that sound project management of the process and structural elements is critical to a successful outcome.

\section{<Figure 3 about here}




\subsection{Scenario Planning as a Creative Process}

Following the argument articulated in section 2 above, we note that creative processes can be seen as varied and stage based, and to contain generic thought-action elements as illustrated in Figure 3. By inspection, the scenario process can be compared with the creative processes of Poincare', Weisburg and Kantor presented in Figure 2. The scenario process is stage based, with thought-action elements. The elements of diagnosis, exploration, scenario building and testing map well onto the phases of the Poincare' model. The interaction of convergent and divergent thought processes is experienced in the scenario process especially in the elements of diagnosis and analysis (convergence) and scenario building and writing (divergent). These thought processes interact in the rich dialogue between participants in the exploration stage. Finally, though Kantor's model is expressly organisational, its features are all reflected in any generic scenario process e.g., idea activation (diagnosis), idea generation (scenario building and writing) and transfer and diffusion (scenario to strategy).

In addition, the scenario process can be mapped tightly onto the West and Farr [7] definition of innovation with its ingredients of 'intentional introduction and application' (diagnosis) of 'ideas...' (exploration, building, writing) that are 'new to the relevant unit of adoption'. In the last 20 years, the majority of scenario processes were new to the unit of adoption. But even if units are familiar with the process, any iteration should produce information, ideas, interpretations and scenarios new to the unit due to the changes in, 
and different impacts of, the external drivers since the previous enactment of the process.

From such inspection, it can be concluded that any SPP that embraces 'novelty and utility' can be interpreted as a creative process and be expected to deliver the wholesome benefits that result from its duration and application. Often, the SPP is seen as creative in its entirety. But, like most creative processes, the reality mixes long periods of mundane activity with short sparks of genial thought as reflected in the words of the film director Ingmar Bergman, "Eight hours of waiting might be rewarded with ten or 12 minutes of real creative work". Moresover, such phases are interspersed with different levels of creativity at different stages within them, as in other artistic pursuits e.g., in writing a novel, composing theme music or painting a landscape.

\section{$<$ Table 1 about here>}

\subsection{Creativity and Dysfunctions Across and Within Individual Stages}

Table 1 shows the typical scenario process activities and provides an assessment of their individual level of creativity. Creativity and its dysfunctions can vary across the stages of the process, within each stage and through a combination of each. For example, in the former, as the higher levels of creativity come towards the end of the process, the beginning takes on a greater significance. The process is information hungry so it is critical to diagnose the issue correctly at the outset and to direct the search for the right data and the signals therein, so that creativity has a fruitful and appropriate platform on which to operate. Mistakes at an early stage can waste the creative activity later. 
However, within the activities of the process, creativity levels can vary widely. For example, data collection can involve routine searches in libraries and archives for extant publications and also include the rich art of conversation in individual or group interviews. The dysfunctions identified below come from case evidence of over 120 scenario projects conducted from the University of St Andrews from 1988 to 2007. They are not portrayed as a comprehensive collection but as the ones that occur often and with damaging consequences.

\subsubsection{Creativity Induced Dysfunctions in the SPP}

\section{Creativity upon Fantasy}

If the data collection activity is de-layered, we find the creative element within interviews varies with the type of interview, whether these are unstructured, semi-structured, structured, group based, active or long. Douglas [30] talked of the 'creative' interview as a special category that challenged the shallow output of conventional approaches to interview questioning and procedures, whose rootings were in the quasi science of rationality and perceived objectivity. Creative interviewing, with its roots in the tradition

of oral history, attempts to capture the 'emotional well springs' of respondents thus capturing deeper thoughts, explanations and dreams:

"Creative interviewing involves the use of many strategies and tactics of interaction, largely based upon an understanding of friendly feelings and intimacy, to optimise co-operative, mutual disclosure and a creative search for mutual understanding". [30, p.25]

In heuristic approaches to scenario planning methods, a variation on Douglas's theme is often used whereby trigger questions (see Table 2) probe a deeper psychology of 
reasoning and reflection and stimulate the desired 'art of strategic conversation'. These questions can be adapted and phrased differently. Van der Heijden [31] encourages playfulness to relax the interviewee by suggesting a 'lighter' wording e.g., 'feel free to explore various unusual avenues'.

But Radcliffe [32] warns that available time, physical location, agency, issue and character of the participant will condition the degree of 'lightness' employed. In some cases, the trigger question approach can stimulate answers that are too creative, taking the interview into incredible realms of fanciful ideas (the dark side). This has to be checked to protect the credibility of the process. Corrections include switching to a more systematic interviewing technique, for example, by using assessments of sectoral trends in political, economic, socio-demographic, technological, legal and environmental trends (the more familiar PESTLE format) or by sending interviewees selected materials beforehand to stimulate a two-way dialogue rather than eliciting just an opinion [33]. Without such countermeasures, the darker side of fantasy creeps into the process's natural agenda. As the latter forms the data platform for later work, which we argue has a higher level of creativity (Table 1), there is a danger of forging a 'double jeopardy' of imposing 'creativity upon fantasy' that might seduce the credulous and carry the day if not checked itself by strong facilitation.

\section{Heightened Expectations and Confusion}


The exploration and scenario building activities are high in creativity and intensity. They take participants into a world of thinking that departs significantly from their normal work life as the focus on two key elements. First, there is a need to evaluate the basic trends from the past, though the present and how these might shape up over the future time horizon of the project. This activity taxes thinking, lifting it from the humdrum of routine line and staff activity into history, social progress, variable complexity and projection. Second, there is a need to examine key uncertainties and to make judgement on how these might play out in an unseen future should they occur at all. This activity stretches thinking, lifting it into the realms of creativity and beyond the taxing of thinking involved in trend extrapolation. For most participants, these activities demand that they activate and utilise those artistic parts of the brain that normally lie dormant for long periods whilst they carry out their daily routines, and thus, for many, it is a process too complex to grasp in such a short time [33]. However, for those capable of understanding fully the scenarios and their implications, it is an exciting phase and one that lifts their imagination to a different plane where they have to deal with both facts and perceptions and the stuff of 'outer space' [4].

This lifting heightens personal expectations and, once the SPP has been completed, participants begin asking questions about a) how the world might be in the future and b) what their job prospects in that world might be and c) their current job routines. Once their lens has been changed and their cognition adjusted by the SPP, this re-evaluation can lead to discontent in the current job (which is seen as irrelevant for the imagined future), low performance, frustration and stress. Hence, their levels of motivation 
become much reduced. These behaviours mirror those identified by [17] on workplace creativity and dysfunction. Inevitably, performance suffers in the absence of strong scenario to strategy communication and engagement.

\section{Pride and Passion}

Progressive senior management and CEOs focussed on the future and encouraging inclusivity and ownership of the strategy process use the SPP as a device to better inform decision making and to test the robustness of their core competencies. The SPP does not work well without their full sponsorship [34] and works well if they play a sensible and not a dominant role [33]. Over time, these senior decision-taking groups will have developed a set of belief systems about how their world works and these will be shared widely. Persistent social interaction leads to the adoption of a common language and 'dictionary of expression' and the creation of social norms and constructs. Moreover, in mature organisations these shared belief systems translate into common visions about what the organisation represents, its operations coding and a particular way that 'we do business around here'. Intelligence about the world around them is interpreted through a strategic kaleidoscope until shaped into a common and acceptable pattern. Amongst other titles, these collective beliefs have been termed 'theories in action' [35], 'dominant logic' [36] and 'interpretative schema' [37] and may be held broadly in an industry strategic group as strong 'recipes' [38]. As Grinyer [39] points out, they are likely to be embedded in the routines that determine day to day behaviour in organisations and, being reinforced by past success, become highly persistent and difficult to change. 
Such beliefs will shape the SPP in their light at the diagnosis activity and it will not confront them directly throughout the first six activities (see Table 1), so a sense of acceptance is generated among senior staff. But the creative elements in the exploration and scenario building activities will begin to question their authenticity, durability and necessity in a slow but increasing manner as the SPP progresses. In particular, in the final scenario to strategy or policy activity, these belief systems can face their major challenge. In interpreting the meaning for current strategy of the future scenarios, senior staff can adopt powerful defensive routines to protect their territory if this 'comfort zone' is placed under duress by the scenarios. In some cases, experience has shown that the CEOs can be 'hoist by their own petard', as the SPP they commissioned suggests the unthinkable in the jettisoning of current strategy. More acute, case examples exist whereby current management styles may not be appropriate for future strategy and even the post of CEO is challenged outright by the SPP that may lead to change at the top. The dysfunction here is not the shining of a powerful beam of light upon current strategy and senior management roles but the detrimental impact on motivation and pride of a skilled executive team or individuals within it. Consequently, existing management can pursue negative behaviour manifest in a rejection of the scenarios as only a set of futures that 'may' happen and the dismissal of the associated new strategies as redundant due to a 'fit of pique' as pride is damaged [40]. Often, senior teams persist in illustrating that their current strategy will work in the face of any scenario. The scenarios are likened to 'disruptive technology' [41] and the organisation is threatened by the usual Luddite-dysfunctions that accompany it. In addition, case evidence suggests 
that there is a risk that credibility is damaged to the point whereby real leadership talent exits the organisation-the 'baby and the bath water' syndrome. In such circumstances, the presence of other stakeholders e.g., respected board members or senior consultants, should be encouraged to intervene and provide fresh insight into the local politics.

\section{Creativity to Excess}

Problematic issues with the SPP are many, varied and well documented. Schoemaker [34] identifies 10 process and 10 content pitfalls and the majority (85\%) of these are driven by a lack of creativity. However, the remainder are the results of too much creativity for the participants of the process. Perceptions of creativity, as novelty and utility, vary between actors in an SPP. Those exposed frequently to worldly issues e.g., members of the strategy staff function, will be relatively comfortable with exposure to surprises and unusual trends and their impact. Line managers with a focus on action orientated operational issues, whose presence is essential if the scenarios are to lead to strategy or policy, may fear aspects of the SPP more. A comfort zone influenced by rational-logical and linear thinking may determine their daily routines. When exposed to the uncertainty, circularity and eclectic nature of worldly issues, orbiting in a system far removed from their conventional space, the comfort zone is challenged seriously. From the beginning, an SPP may seem like an irrelevant exercise that is easy to approach in a sceptical way. They will identify with the earlier data collection and data base phases and good facilitation should ensure a stimulating and enjoyable experience when it comes to the creative phases of exploration, scenario building and scenarios to strategy. 
Because such participants can perceive of a process, action or narrative as more creative than those used to such a process, a number of dysfunctions can set in. First, the more creative phases of the SPP can be seen as 'just a bit of fun' [42] being perceived as so far removed from their mental model of reality as to be close to fantasy. In particular, when they are asked to live and work out life in each future scenario, their experiences can be frightening and distressing leading to either a rejection of the SPP as irrelevant or its acceptance as just a team building game to be ignored once back in the office. But for some, memories of their play in the future can depress them once back in their job in the present and make their role feel insignificant, lowly and detached from how they have understood the future might be.

Second, no matter what their experience of the SPP, line managers taken off their routine tasks to undertake a futures exercise, have to return to their tasks on 'Monday morning'. The excitement of dealing with creativity, narrative, imagination, dream and intuition soon fades once the routine tasks are taken up again. These can cause a return of the prior 'comfort zone' and its automatically triggered 'business as usual' procedure. It is tough to link the scenarios to this everyday world, as they are perceived as too creative, too distant and too long into the future. Such incongruity leads to rejection of the SPP as having no meaning, relevance or sense making virtues in their local domain-without trust they lose credibility [43]. Something seen as too creative, even when the scenarios are at varying levels of normative neutrality, will struggle to compel action and make a real breakthrough at task level. 
Third, in grappling with numerous uncertainties and pre- determined trends, multiple scenarios may be built to cope with the variety of potential futures. The challenge is to contain these into a comprehensive and comprehensible number. Any more than four or five scenarios taxes cognition and are difficult to translate into informed strategy. But when increasing complexity is coupled with powerful and diverse political views of senior managers, the creativity required could move into excess and larger numbers of scenarios are built to cope with the converging pressures. There is a trade off between creativity and effectiveness i.e., the more the creativity in numbers of scenarios constructed the less likely that strategy or policy can be built to reflect their variety. Finally, scenarios are subjected to several critical tests, including that for internal consistency. The credibility of scenarios demands that the individual elements of the stories link logically to each other, from the past, through the present and to the future and correlate well with each other. Equally, scenarios must contain a 'wow' factor inducing great surprise on an initial read or presentation as if combining the surprise with the credible in 'wow... wait a minute, that could happen'. Another trade-off is presented such that too much creativity in the search for the 'wow' factor causes internal consistency to be stretched as variables enter the scenarios that challenge their internal credibility e.g., full employment together with zero inflation. The more creativity that is used in the search for surprise can result in less consistency and credibility in the scenarios. These latter trade-offs impose 'natural' limits on the amount of creativity that can be used in the SPP.

$<$ Insert Table 3 about here> 


\subsection{Concluding Comments}

This paper has sought to investigate the dysfunctional effects of creativity in a heuristic SPP. After identifying definitions of creativity and the creative process from the areas of social psychology and the cultural industries, we asked whether the SPP was a creative process. Using comparative analysis, it was argued that the SPP passed the criteria and content for a creative process. Further, using the SPP as a vehicle, the paper identified areas where its creativity could have dysfunctional effects, in particular in the areas of creativity versus fantasy, expectations and confusion, pride and passion and excess. Trade offs for creativity with greater numbers of scenarios and effective strategy translations and for creativity and surprise and logical consistency were revealed. Clearly creativity does not live in isolation from the context or content of a strategy process and the SPP is no exception. These factors imbue each process with a unique mix of perceptions, belief systems, urgencies and power battles. So the extent of the dysfunctional aspects of creativity will be contingent upon these 'shaping' factors. For many, the presence of creativity will be both necessary and virtuous, shedding new light on ways of seeing and doing things. For others, its dysfunctional effects may be great enough to render it neutral at best and harmful at worst.

For a heuristic based SPP, our analysis points to four options:

a) Accept the creativity of this type of SPP as it informs the strategy or policy process and the dysfunctional effects that can accompany it, managing their implications as described in Table 3. This approach requires 'innovation within' the existing SPP (as illustrated in Figure 3 and Table 3) with the expectation that such 
correcting factors may limit the degree of dysfunction through amelioration and produce a creative, yet robust, outcome.

b) Accept that the majority of SPPs are derived from a common family and that there will always be a potential for creativity-driven dysfunction to damage the process and outcome. This approach reflects a 'status quo' option and assumes that any correcting factors will fail to ameliorate the potential excesses of creativity and so the extra effort and design required incorporating them into the SPP is unjustified.

c) Conduct a major re-engineering of the heuristic SPP from first principles (e.g., by addressing the complexities of the modern world with a focus on the design of efficient, short run scenarios capable of fast metamorphosis for the next brief time period) or fortify the existing SPP with other approaches to building scenarios (e.g., with the methodologies of the French La Prospective School [25] [44]) to produce a new variety of foresight tool. These approaches would require conclusive proof that the dysfunctional elements were so great as to be incapable of amelioration and would negate any practical utility for the outcomes of the existing SPP. At present, that conclusive proof is itself 'prospective'.

d) Accept the need to reject a heuristic based SPP as a foresight tool and attempt to deal with increasing complexity by falling back on more traditional forecasting based options e.g., quantitative based systems models. This stark approach accepts fully the need to reject the role that 'intuitive logics' or 'disciplined imagination' can play in the foresight projects. The internal messiness of these 
processes may not be capable of making sense of the external messiness for which they were designed.

From its increasing popularity among organisations and agencies, it appears that the use of a heuristic SPP has delivered greater benefits than disruptions. Moreover, these are early days in the investigation of the potential dysfunctional aspects of creativity in the heuristic SPP. Hence, leaving the current SPP (Figure 3) in tact (b) or innovating within it (Table 3) (c) would seem to be better choices than either an immediate rejection (d) or a major re-engineering or fortification project (c) as far as productivity in foresight studies goes. Embracing creativity at the heart of the heuristic SPP accepts that all creative acts have the potential for dysfunctional consequences and these may have to be managed better in a refined SPP than they have been in the past.

The definition of scenario planning in this study (op cit) emphasised its use as an intervention in the field of strategic management, the provider of plausible future states and the practical means of providing for them. Its power as an intervention tool is strengthened by awareness of potential dysfunctions embedded within its design and the danger signals that should indicate whether corrective treatment might be required. As the provider of maps of how the future terrain might look, counterbalancing innovations within its existing process should ameliorate any excesses of embedded creativity and allow the preservation of the essential plausibility of those maps. As a foresight tool that informs strategy and policy in a practical way its capability is improved through innovations in the process that keep grounded rather than airborne. 
References

1. Anderson, N., C.K.W. De Dreu, and B.A. Nijstad, The routinization of innovation research: a constructively critical review of the state-of-the-science. Journal of Organizational Behavior, 25 (2004), p. 147-173.

2. Wittgenstein, L., Philosophical Investigations. Upper Saddle River, NJ: Prentice Hall. (1999)

3. Wack, P., Scenarios: Uncharted Waters Ahead. Harvard Business Review, 63 (5) (1985a), p. 72-79.

4. Wack, P., Scenarios: Shooting the Rapids. Harvard Business Review, 63(6) (1985b), p. 139-150.

5. Quine, W.V., The Philosophy of Logic. 2nd ed. Oxford: Oxford University Press. (1970), (1970)

6. Bilton, C. Creative Management: from individual talent to collective process. in Creative Industries Workshop, Creative Industries Observatory. (2007)

7. West, M.A. and J.L. Farr, Innovation at work, in Innovation and creativity at work, M.A. West and J.L. Farr, Editors. Wiley: Chichester, England. (1990) p. 1-13.

8. King, N., Modeling the innovation process: an empirical comparison of approaches. Journal of Occupational and Organizational Psychology, 65 (1992), p. 89-100.

9. Anderson, N. and R.M. Gasteiger, Helping creativity and innovation thrive in organizations: Functional and dysfunctional perspectives, in Research Companion to the Dysfunctional Workplace: Management Challenges and Symptoms, J. Langan-Fox, C.L. Cooper, and R.J. Klimoski, Editors. Edward Elgar Publishing: Cheltenham. (2007),

10. Boden, M.A., What is Creativity?, in Dimensions of Creativity, M.A. Boden, Editor. MIT Press: Cambridge, MA. (1994) p. 75-117.

11. De Bono, E., Lateral Thinking: Creativity Step by Step. New York, NY: Harper \& Row. (1970)

12. King, N. and N. Anderson, Managing innovation and change: A critical guide for organizations. London: Thompson. (2002)

13. Woodman, R.W., J.E. Sawyer, and R.W. Griffin, Toward a Theory of Organizational Creativity. Academy of Management Review, 18(2) (1993), p. 293-321. 
14. Scott, S.G. and R.A. Bruce, Determinants of Innovative Behavior: A Path Model of Individual Innovation in the Workplace. Academy of Management Journal, 37(3) (1994), p. 580-607.

15. Rank, J., V.L. Pace, and M. Frese, Three Avenues for Future Research on Creativity, Innovation, and Initiative. Applied Psychology, 53(4) (2004), p. 518-528.

16. James, K., K. Clark, and R. Cropanzano, Positive and negative creativity in groups, institutions, and organizations: A model and theoretical extension. Creativity Research Journal, 12 (1999), p. 211-226.

17. Janssen, O., E. van de Vliert, and M. West, The bright and dark sides of individual and group innovation: A Special Issue introduction. Journal of Organizational Behavior, 25 (2004), p. 129-145.

18. Livingstone, L.P., D.L. Nelson, and S.H. Barr, Person-environment fit and creativity: An examination of supply-value and demand-ability versions of fit. Journal of Management, 23 (1997), p. 119-146.

19. Townsend, A., S. DeMarie, and A.R. Hendrickson, Virtual teams: Technology and the workplace of the future, in The human side of managing technological innovation: $A$ collection of readings, R. Katz, Editor. Oxford University Press: New York. (2004)

20. De Dreu, C.K.W., When too little or too much hurts: evidence for a curvilinear relationship between task conflict and innovation in teams. Journal of Management, 32 (2006), p. 83-107.

21. Sackett, P.R. and C.J. DeVore, Counterproductive behaviors at work, in Handbook of industrial, work, and organizational psychology, N. Anderson, et al., Editors. Sage: London / New York. (2001) p. 145-199.

22. Kantor, R.M., The Change Masters: Innovation and Entrpreneurship in the American Corporation. New York, NY: Simon \& Schuster. (1983)

23. Weisberg, R.W., Creativity: Beyond the myth of genius. New York, NY: Freeman. (1993)

24. Poincare, H., The Foundations of Science: Science and Hypothesis, The Value of Science, Science and Method. Washington: University Press of America. (1982)

25. de Jouvenel, B., The Art of Conjecture. London: Weidenfeld \& Nicolson. (1967)

26. Kahn, H. and A.J. Wiener, The Year 2000: A framework for speculation on the next thirty-three years. New York: MacMillan. (1967)

27. Hart, G. and W.B. Rudman, 21st Century Terrorism and National Security. US Commission on National Security: Washington, DC. (2000)

28. National Commission on Terrorist Attacks Upon the United States, The 9/11 Commission Report: Final Report of the National Commission on Terrorist Attacks Upon the United States. W. W. Norton \& Company: New York / London. (2004)

29. MacKay, R.B. and P. McKiernan, The role of hindsight in foresight: refining strategic reasoning. Futures, 36 (2003), p. 161-179.

30. Douglas, J., Creative Interviewing. Beverly Hills, CA: Sage. (1985)

31. van der Heijden, K., Scenarios: The Art of Strategic Conversation. Chichester: Wiley. (1996) p 320.

32. Radcliffe, D., Technological and Pedagogical Convergence between Work-based and Campus-based Learning. Educational Technology \& Society, 5(2) (2002) 
33. Moyer, K., Scenario Planning at British Airways - A case study. Long Range Planning, 29(2) (1996), p. 172-181.

34. Schoemaker, P.J.H., Twenty Common Pitfalls in Scenario Planning, in Learning from the Future, L. Fahey and R. Randall, Editors. (1998), John Wiley \& Sons. p. 422-431.

35. Argyris, C. and D. Schon, Organisational learning: A theory of action perspective. Reading, MA: Addison Wesley. (1978)

36. Prahalad, C.K. and R. Bettis, The Dominant Logic: A new logic between diversity and performance. Strategic Management Journal, 7 (1986), p. 485-501.

37. Bartunek, J.M., The dynamics of personal and organisational reframing, in Paradox and Transformation: Toward a Theory of Change in Organization and Management, R.E. Quinn and K.S. Cameron, Editors. (1988), Ballinger: Cambridge, MA. p. 137-162.

38. Spender, J.C., Industry recipes: an inquiry into the nature and sources of management judgement. London: Basil Blackwell. (1989)

39. Grinyer, P., A cognitive approach to group strategic decision taking: discussion of evolved practice in the light of received research results. Journal of the Operational Research Society, (2001), 51.

40. Whittington, R., Completing the Practice Turn in Strategy Research. Organization Studies, 27(5) (2006), p. 613-634.

41. Christensen, C.M., The Innovator's Dilemma. London: Harper Collins. (2003)

42. Mante-Meijer, E., P. van der Duin, and M. Abeln, Fun with scenarios. Long Range Planning, 31(4) (1998), p. 628-637.

43. Selin, C., Trust and the illusive force of scenarios. Futures, 38(1) (2006), p. 1-14.

44. Godet, M., (2001), Creating Futures: scenario planning as a strategic management tool, Economica, London. 


\section{Figure 1: Process Models}

(From Journals AMR, AMJ, AP, JoB)

Input $\longrightarrow$ Transformation $\longrightarrow$ Output

Antecedent

Variables

$$
\text { AVs } \rightarrow C \longrightarrow \begin{aligned}
& \text { Moderating } \\
& \text { Variables }
\end{aligned} \rightarrow \mathrm{I} \rightarrow \text { Output }
$$

$$
\text { AVs } \rightarrow C \rightarrow M V s \rightarrow I \rightarrow \text { Output } \ \begin{aligned}
& \text { Benefits } \\
& \text { Costs }
\end{aligned}
$$

(Where $C=$ Creativity and I=Innovation) 


\section{Figure 2: Creative Processes}

(adapted from Bilton, 2007)

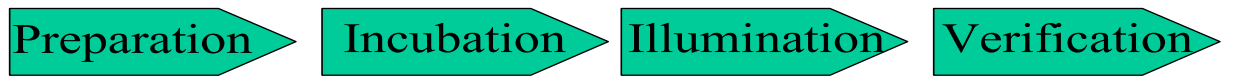

(Poincare, 1982)

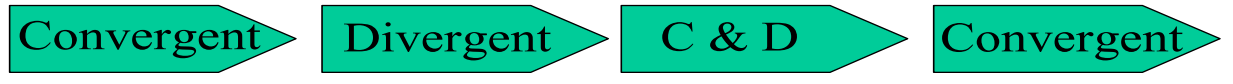

(Weisberg, 1993)

Innovation

Activation

Idea

Generation

(Kantor, 1988)
Coalition

Building
Transfer \&

Diffusion 
Figure 3: The Scenario Planning Process

(Source: McKiernan, 2007)

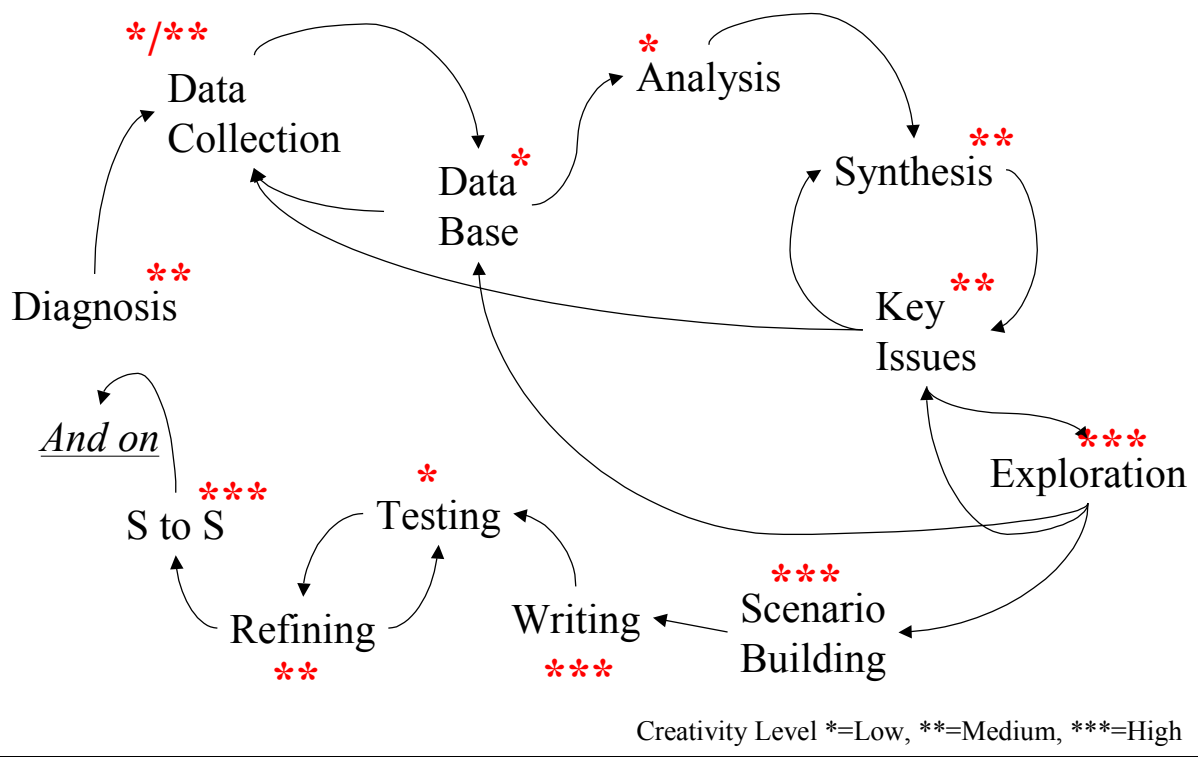

Table 1: A Generic, Heuristic- based Scenario Planning Process 


\begin{tabular}{|c|c|c|}
\hline Diagnosis & Small Team Dialogue & Low-Medium \\
\hline Data Collection & Archive, Interview & Low-Medium \\
\hline Data Base & Collation \& Recording & Low \\
\hline Analysis & Classify \& Order & Low \\
\hline Synthesis & Map \& Cluster & Medium \\
\hline Key Issues & Debate & Medium \\
\hline Exploration & Workshop Dialogue & High \\
\hline Scenario Building & Soft Systems Modelling & High \\
\hline Writing & Draft Narrative & High \\
\hline Testing & Tests on Content & Low \\
\hline Refining & Finessing of Narrative & Medium \\
\hline Scenarios to Strategy & Workshop Dialogue & High \\
\hline \multicolumn{3}{|c|}{$\begin{array}{l}\text { Notes: } \\
\text { a) The Activity elements are shown in a linear order for ease of presentation. In reality, they do no follow this order } \\
\text { necessarily and there is much iteration within it (see Figure 1). } \\
\text { b) The Creativity weightings are based upon experiential observation of a team of scenario facilitators at the University of St } \\
\text { Andrews, based upon over } 150 \text { scenario projects over a } 20 \text {-year period and tested with scenario experts at the Third } \\
\text { International Foresight Conference at the University of Strathclyde in September } 2007 \text {. }\end{array}$} \\
\hline
\end{tabular}




\section{Table 2: Seven Trigger Questions}

1 What do you think are the critical issues for the future?

2 If things went well, what would you see as a desirable outcome?

3 If things went wrong, what factors would you worry about?

4 How would internal systems have to change to bring about the desired outcome?

5 What are the significant past events that have produced the current situation?

6 What do you see as the priority actions, which should be carried out soon?

7 What would you like to have done if all the constraints were removed?

Original Source: Amara \& Linpinsky, 1983 with adaptations from Shell and St Andrews

Table 3: Stages of the SPP, Creativity Induced Dysfunctions and Danger Signals 


\begin{tabular}{|l|l|l|l|}
\hline SPP Activity(s) & Cl Dysfunction & Implication & Danger Signals \\
\hline Data Collection & Creativity upon Fantasy & $\begin{array}{l}\text { Caution in personal } \\
\text { interviewing, balancing } \\
\text { 'lightness' with reality }\end{array}$ & $\begin{array}{l}\text { Excessive play } \\
\text { Absence of data } \\
\text { Absence of expert } \\
\text { opinion }\end{array}$ \\
\hline $\begin{array}{l}\text { Exploration \& } \\
\text { Scenario Building }\end{array}$ & $\begin{array}{l}\text { Heightened Expectations } \\
\text { \& Confusion }\end{array}$ & $\begin{array}{l}\text { Care to design good } \\
\text { communications in the } \\
\text { scenario to strategy } \\
\text { engagement }\end{array}$ & $\begin{array}{l}\text { Random team } \\
\text { selection } \\
\text { Repetitive critique of } \\
\text { the present }\end{array}$ \\
\hline Diagnosis and SPP & Pride \& Passion & $\begin{array}{l}\text { Sensitive but firm dialogue } \\
\text { and guidance at Diagnosis, } \\
\text { inclusion of respected } \\
\text { externals throughout SPP }\end{array}$ & $\begin{array}{l}\text { CEO dominance } \\
\text { CEO omnipresence } \\
\text { Excessive use of same } \\
\text { metaphor } \\
\text { Critique of SPP }\end{array}$ \\
\hline $\begin{array}{l}\text { Key Issues, Exploration, } \\
\text { Scenario Building }\end{array}$ & Creativity to Excess & $\begin{array}{l}\text { Design balanced teams, } \\
\text { careful management of } \\
\text { expectations and policies } \\
\text { for re-entry, strong } \\
\text { facilitation during building } \\
\text { \& testing stage }\end{array}$ & $\begin{array}{l}\text { Excessive joviality } \\
\text { Absence of critique of } \\
\text { the present } \\
\text { Multiple scenarios } \\
\text { Overt focus on } \\
\text { designing surprise }\end{array}$ \\
\hline
\end{tabular}

